\title{
Vocal Pathways Modulate Efferent Neurons to the Inner Ear and Lateral Line
}

\author{
Matthew S. Weeg, Bruce R. Land, and Andrew H. Bass \\ Department of Neurobiology and Behavior, Cornell University, Ithaca, New York 14853
}

\begin{abstract}
All sonic vertebrates face the problem of sound production interfering with their ability to detect and process external acoustic signals, including conspecific vocalizations. Direct efferent inputs to the inner ear of all vertebrates, and the lateral line system of some aquatic vertebrates, represent a potential mechanism to adjust peripheral sensitivity during sound production. We recorded from single efferent neurons that innervate the inner ear and lateral line in a sound-producing teleost fish while evoking fictive vocalizations predictive of the temporal features of natural vocalizations. The majority of efferent neurons showed an increase in activity that occurred in-phase with modulations in the fine temporal structure of the fictive vocalizations. Many of these neurons also showed a decrease in activity at fictive vocal offset. Efferents to the sacculus, the main auditory end organ, showed features especially well adapted for maintaining sensitivity to external acoustic signals during sound production. These included robust phase locking of efferent activity to each cycle of a fictive vocalization and a long-duration rebound suppression after each fictive vocalization that could provide a rapid, long-lasting period of sensitization to external acoustic stimuli such as the call of a conspecific. These results suggest that efferent activation by the vocal motor system can directly modulate auditory sensitivity to self-generated sounds and maintain sensitivity to ongoing external sounds. Given the conserved organization of the auditory efferent system across vertebrates, such mechanisms may be operative among all sonic vertebrates.
\end{abstract}

Key words: vocalization; audition; lateral line; temporal modulation; teleost; hearing

\section{Introduction}

Sound-producing animals are faced with the challenge of stimulating their own auditory systems with the signals they produce, a phenomenon known generally as sensory reafference (von Holst and Mittelstaedt, 1950). Reafferent signals potentially interfere with the reception of external sounds by either masking these sounds during, or reducing the sensitivity of the auditory system immediately after, sound production. Filtering and removal of these signals at one or more levels of the auditory system is therefore crucial to maintain sensitivity to external sounds (Poulet and Hedwig, 2002 and references therein).

One mechanism for adjusting peripheral auditory sensitivity in vertebrates is the middle ear response. During either sound production or stimulation by intense external sounds, contraction of middle ear muscles limits movement of the middle ear bones, which reduces the transmission of acoustic energy to sensory hair cells in the cochlea (Grassi et al., 1988; Yost, 2000). Although effective, the middle ear response represents a highly derived adaptation with structural requirements (i.e., middle ear

Received Jan. 4, 2005; revised May 10, 2005; accepted May 10, 2005.

This research was supported by National Institutes of Health/National Institute on Deafness and Other Communication Disorders Grant DC00092. We thank B. Carlson, H. Farris, and C. Hopkins for providing critical feedback on this manuscript.

Correspondence should be addressed to Andrew H. Bass, Department of Neurobiology and Behavior, Seeley G. Mudd Hall, Cornell University, Ithaca, NY 14853. E-mail: ahb3@cornell.edu.

M. S. Weeg's present address: Department of Biology, Colorado State University, Fort Collins, CO 80523. E-mail: mwerg@lamar.colostate.edu.

D0I:10.1523/JNEUROSCI.0019-05.2005

Copyright $\odot 2005$ Society for Neuroscience $\quad$ 0270-6474/05/255967-08\$15.00/0 bones and muscles) that preclude it from being a gain-control mechanism across vertebrates. In contrast, all vertebrates possess central efferent neurons that modulate the activity of the peripheral auditory system (Roberts and Meredith, 1992), thereby providing a common mechanism by which the vocal motor system could adjust inner ear sensitivity during sound production.

Male plainfin midshipman fish, Porichthys notatus, produce vocalizations during courtship and agonistic interactions that potentially interfere with the sender's ability to detect either conspecific vocalizations or other salient acoustic signals (for review, see Bass et al., 1999). Anatomical studies have shown a connection between the vocal motor system and an efferent nucleus in the rostral medulla that innervates inner ear end organs and mechanosensory lateral line receptor organs (Bass et al., 1994). Given the encoding of vocal parameters by both the auditory (for review, see Bass and McKibben, 2003) and lateral line (Weeg and Bass, 2002) systems in midshipman fish, we hypothesized that the efferent system is activated during sound production, leading to changes in the neuronal activity of both inner ear and lateral line primary afferents. Midshipman and the closely related toadfish are particularly well suited to address this issue because fictive vocalizations, i.e., rhythmic vocal motor output predictive of the temporal features of natural vocalizations, are readily elicited (Bass and Baker, 1990), and the efferent neurons project to their target end organs in bundles that are anatomically distinct from the primary afferent nerves, thereby providing a pure population of efferent fibers for neuronal recordings (Highstein and Baker, 1986). 
We report, to our knowledge, the first demonstration of central vocal motor-induced modulation of efferents to the lateral line and inner ear that parallels the fine temporal structure of vocalizations. This includes efferents to the primary auditory end organ, the sacculus (Popper and Fay, 1999; Bass and McKibben, 2003). Given the similarities in the structure and operation of the auditory systems of teleosts and other vertebrates (Popper and Fay, 1999), it seems likely that such mechanisms are also operative among other sonic tetrapods that need to solve sensory reafference problems.

\section{Materials and Methods}

Midshipman fish have two adult male reproductive morphs known as type I and type II males (Bass and McKibben, 2003). We focused our studies on type I males because of their dynamic vocal repertoire; they alone acoustically court females and generate agonistic growls and grunt trains during the breeding season, whereas type II males and females infrequently produce isolated grunts during nonreproductive contexts. Nineteen type I male midshipman (35.0-76.2 g; standard length, 13.7$17.9 \mathrm{~cm}$ ) were used in this study. Animals were collected from nest sites in Tomales Bay, CA, or by trawl in Monterey Bay, CA, and were maintained in artificial seawater tanks at $12-16^{\circ} \mathrm{C}$ on a regular diet of goldfish. All experiments were conducted under the guidelines of the Cornell University Institutional Animal Care and Use Committee and the National Institutes of Health.

Before surgery, fish were anesthetized by immersion in $0.025 \%$ ethyl $p$-amino benzoate (Sigma, St. Louis, MO) in artificial seawater. The cranium was entered dorsally to expose the midbrain, branches of the auditory nerve, and the ventral occipital nerve roots that innervate sonic muscles attached to the swim bladder (Bass et al., 1994). Excess fluid was blotted from the cranial cavity and replaced with a clear, inert fluid (Fluorinert; 3M, St. Paul, MN). Fish were then transferred to a $32-\mathrm{cm}-$ diameter tank filled with artificial seawater $\left(12-16^{\circ} \mathrm{C}\right)$ and placed onto a lucite head holder. A tube inserted into the fish's mouth provided recirculated seawater across the gills. Animals were given intramuscular injections of fentanyl $(1.0 \mathrm{mg} / \mathrm{kg}$ body weight; Sigma) for analgesia and pancuronium bromide $(0.5 \mathrm{mg} / \mathrm{kg}$ body weight; Astra Pharmaceutical Products, Westborough, MA) for immobilization, which rendered both sonic and nonsonic muscles inactive during the course of the experiments. The tank rested on a vibration isolation table inside an acoustic isolation chamber (Industrial Acoustics, New York, NY).

Insulated tungsten electrodes ( $125 \mu \mathrm{m}$ diameter; $20 \mu \mathrm{m}$ exposed tips; $5 \mathrm{M} \Omega$ impedance; A-M Systems, Carlsborg, WA) delivered eight electrical pulses $(0.1 \mathrm{~ms}$ pulse duration; $300 \mathrm{~Hz})$ to localize vocally active sites within the midbrain. Fictive vocalizations were monitored using paired silver ball electrodes (50-100 $\mu \mathrm{m}$ diameter) insulated with Teflon except at the tips. One electrode was placed intracranially on a ventral occipital nerve root, and the other was placed in the musculature surrounding the cranium. The synchronized output of sonic motor neurons, the sonic motor volley (Fig. 1A), was amplified, filtered through $300-5000 \mathrm{~Hz}$ (A-M Systems 1700), and digitized (AD1, System II; Tucker-Davis Technologies, Alachua, FL) onto a personal computer running commercial data acquisition software (Brainware; Tucker-Davis Technologies). Preliminary experiments suggested that electrical stimulation of the vocal motor system caused a change in efferent activity that was independent of vocal output. To avoid this confounding effect, the tungsten electrode was replaced with a glass micropipette (tip diameter $\sim 20 \mu \mathrm{m}$ ) filled with $0.1 \mathrm{M}$ glutamate ( $\mathrm{L}$-glutamic acid; Sigma) in teleost Ringer's solution ( $\mathrm{pH}$ 8.0) once a vocally active site was found. Glutamate injections did not cause changes in efferent activity independent of vocal output (see Results).

Extracellular potentials were recorded from single efferent neurons using glass micropipettes filled with $4 \mathrm{M} \mathrm{NaCl}$. Electrodes were placed on the surface of the nerve and advanced using an hydraulic microdrive (David Kopf Instruments, Tujunga, CA). Signals were preamplified using a custom built preamplifier, amplified and filtered through 300-3000 $\mathrm{Hz}$ (P15; Grass Instruments, West Warwick, RI), and digitized (AD1; Tucker-Davis Technologies) onto a personal computer running com-

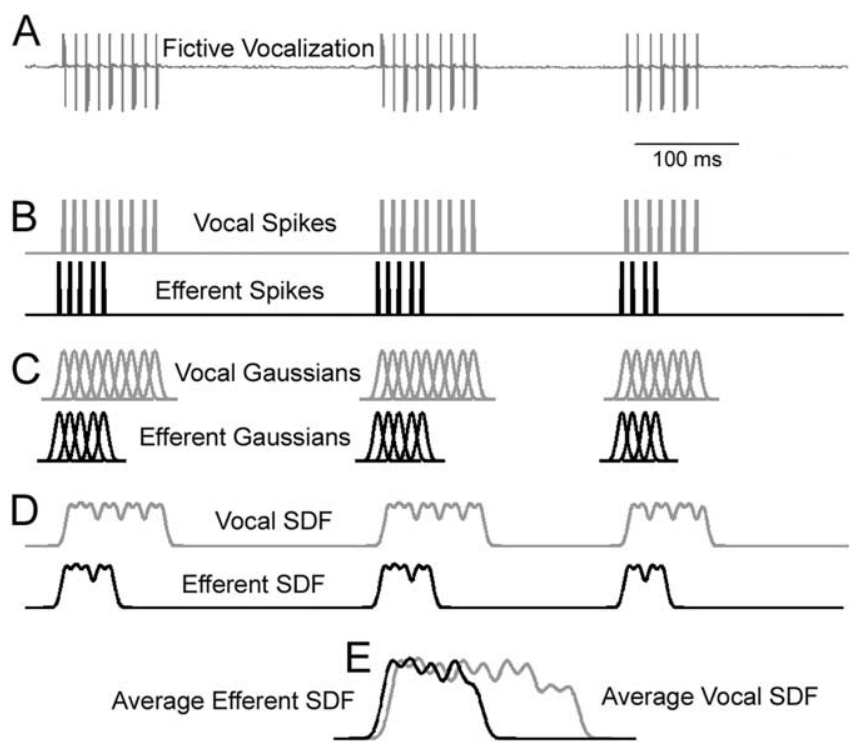

Figure 1. Schematic overview of methods used to construct SDFs. Data shown are representative of responses. With the exception of the fictive vocalizations shown in $A$, the data are computer synthesized for simplicity of presentation. $\boldsymbol{A}$, Synchronized output of the ventral occipital nerve root after glutamate injections into a vocal site in the midbrain. The temporal properties of the sonic motor volley (the fictive vocalization) that is predictive of the basic features of natural vocalizations matches the synchronized, oscillatory-like activity of an expansive pacemaker-motor neuron circuit that extends throughout the caudal hindbrain and rostral spinal cord (Bass and Baker, 1990). B, Data acquisition software captured spike times of compound vocal potentials (gray, top traces) and efferent action potentials (black, bottom traces). C, These were then convolved with a Gaussian window function, which gave a smooth estimate of spike rate. $\boldsymbol{D}$, Combining the individual Gaussians allowed us to automatically detect the onset of fictive vocalizations. $\boldsymbol{E}$, Aligning all individual fictive vocalizations on their onset and averaging both the vocal and associated efferent activity produced SDFs that represented the average change in efferent activity during fictive vocalizations. The actual data permitted finer temporal resolution than the Gaussian simulation shown here. For details, see Materials and Methods.

mercial data acquisition software (Brainware; Tucker-Davis Technologies). Visually identified action potentials were discriminated using a window discriminator within Brainware. After isolation of a single efferent neuron, glutamate was picospritzed into the vocally active midbrain site using 10-500 ms, 20-40 psi air pulses (1050 ms interpulse interval) until fictive vocalizations began. Glutamate delivery was then stopped, and fictive vocalizations typically continued for several minutes. Ongoing efferent activity was collected until fictive vocal activity stopped or recordings became unstable. Recordings of efferent and fictive vocal activity were synchronized in time so that the temporal relationship between the two could be examined (Fig. 1B).

At the end of each experiment, midbrain stimulation sites were marked by either electrolytic lesions or iontophoretic injection $(+3.0$ $\mu \mathrm{A}, 2 \mathrm{~min})$ of $10 \%$ dextran tetramethylrhodamine (10 kDa; Molecular Probes, Eugene, OR) in 0.1 м PBS, 10\% Alexa Fluor 546 (10 kDa; Molecular Probes) in $0.1 \mathrm{~m}$ PBS, or $5 \%$ Neurobiotin (Vector Laboratories, Burlingame, CA) in $3 \mathrm{M} \mathrm{KCl}$. Fish were killed either immediately (lesions and fluorescent tracer injections) or after an $8 \mathrm{~h}$ survival period (biotin injections) by transcardial perfusion of heparinized teleost Ringer's solution, followed by $4 \%$ paraformaldehyde in $0.1 \mathrm{~m}$ phosphate buffer (PB). Glutaraldehyde (1\%) was added to the fixative for brains injected with biotin. Brains were removed, postfixed for $1 \mathrm{~h}$ in fixative, and stored in $0.1 \mathrm{M}$ PB. Brains were placed in $30 \%$ sucrose in $0.1 \mathrm{~m} \mathrm{~PB}$ overnight and sectioned frozen $(50 \mu \mathrm{m})$ on a sliding microtome in the transverse plane. Biotin-injected brains were processed to visualize the label (Weeg and Bass, 2000).

Either $5 \%$ Neurobiotin or $10 \%$ dextran biotin $(10 \mathrm{kDa}$; Molecular Probes) in $3 \mathrm{M} \mathrm{KCl}(+3.0 \mu \mathrm{A}, 3-10 \mathrm{~min})$ was iontophoresed into the fiber bundles from which we recorded to verify that they contained the axons 
of central efferent neurons. After survival times of $8-15 \mathrm{~h}$, fish were perfused with heparinized teleost Ringer's solution, followed by $4 \%$ paraformaldehyde/ $1 \%$ glutaraldehyde in $0.1 \mathrm{M}$ PB. Brains were then sectioned frozen and processed to visualize the label (Weeg and Bass, 2000). We iontophoresed either $10 \%$ dextran tetramethylrhodamine or $10 \%$ Alexa Fluor 546 in 0.1 м PBS into the efferent fiber bundles to identify their targets. After $6 \mathrm{~h}$ survival, fish were perfused with heparinized teleost Ringer's solution, followed by $4 \%$ paraformaldehyde in $0.1 \mathrm{M}$ PB. All inner ear end organ nerves and sensory epithelia, along with the anterior and posterior lateral line nerves, were removed and cleared through an ascending series of glycerin.

To analyze the temporal relationship between fictive vocalizations and efferent activity, we constructed spike density functions (SDFs) (Paulin, 1992; Szucs, 1998) by convolving each efferent and compound vocal action potential with a Gaussian window function (Fig. 1C). We used SDFs because straight binning caused aliasing inconsistencies in spike rate estimation and the times at which the rates changed (for a more general discussion of the advantages of using SDFs for spike train analysis, see Szucs, 1998). Because we were interested in the detailed relationship of efferent spike times relative to the onset time of fictive vocalizations, determining fictive vocal onset time was important. There was a tradeoff in determining the width of the Gaussian to use. A wide Gaussian makes a very smooth estimate of the spike rate but obscures the rise time estimate when spike rate changes suddenly at the start of a fictive vocalization. A narrow Gaussian does not adequately smooth across spikes to allow reliable detection of a burst. To automatically detect the onset of fictive vocalizations, we chose a Gaussian with a width that dropped the average change in SDF between adjacent vocal spikes to $\sim 10 \%$ of the peak SDF (Fig. 1D). This choice gave a time resolution of a few milliseconds. The start time of a fictive vocalization was then identified at the $50 \%$ rise point of the SDF. Once the fictive vocal start time was determined, all efferent spike times could be measured with respect to the fictive vocal start time. The fictive vocalizations were then aligned at the onset and averaged, and the efferent activity associated with each fictive vocalization was averaged (Fig. $1 E$ ). This resulted in a single SDF containing the average fictive vocal and efferent activity, which provided an estimate of the average efferent activity at the onset of the fictive vocalizations. Alternatively, we could detect the $50 \%$ point on the falling edge of a fictive vocalization to obtain the ending time and analyze the average efferent activity at fictive vocal offset. With the estimate of onset and offset times in hand, we could then make new SDFs with higher time resolution (by decreasing the width of the Gaussian function) to more closely examine the temporal relationship between efferent spikes and compound vocal spikes.

Because of the constraints of the experimental paradigm, we were unable to demonstrate a change in primary afferent activity to test the hypothesis that vocal-induced changes in efferent activity affects auditory and lateral line sensitivity. As explained above, we administered paralyzing doses of pancuronium bromide to prevent both the fish from moving and the sonic muscles from contracting during vocal motor volleys. The former is necessary to obtain stable neuronal recordings, whereas the latter is necessary to ensure that the efferent effect did not result from auditory feedback. As an acetylcholine antagonist, however, the levels of pancuronium bromide necessary to achieve immobilization apparently blocked the cholinergic receptors on the hair cells and/or primary afferents, thus eliminating the efferent influence on primary afferent activity (Furukawa, 1981). Attempts to conduct these experiments on anesthetized animals were unsuccessful, because anesthetic severely compromises both vocal and auditory activity in midshipman fish (M. Kittelberger, J. Sisneros, and A. Bass, unpublished observations).

\section{Results}

Stimulation sites were localized within the caudal midbrain vocal-acoustic complex (mVAC), ventral to the torus semicircularis (Fig. $2 \mathrm{~A}$ ); biotin injections labeled the descending vocal system in a pattern consistent with that reported previously (Goodson and Bass, 2002). Glutamate injections into this area elicited a rhythmic motor output, the fictive vocalization (Fig. $1 A$; the du-
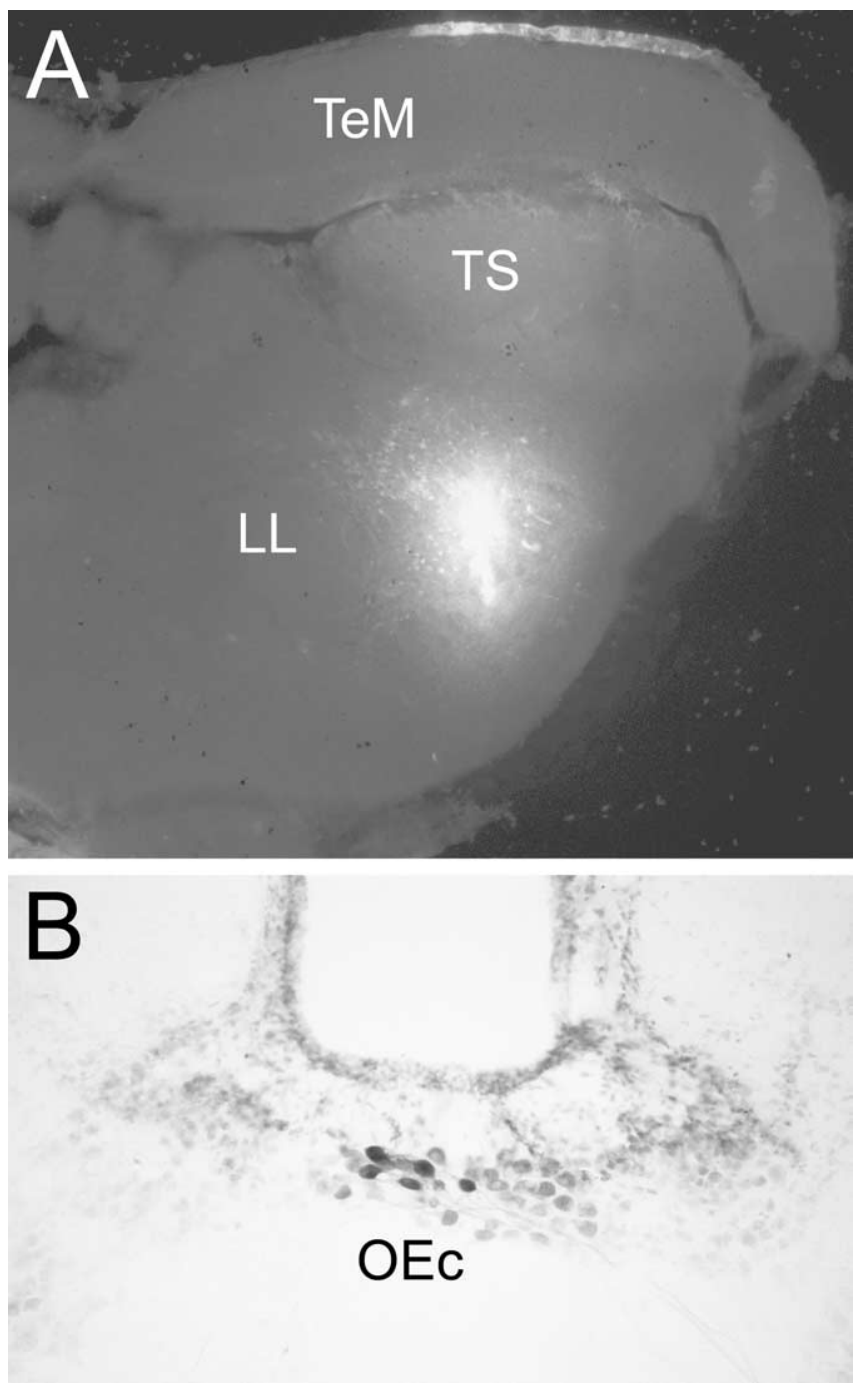

Figure 2. A, Fluorescent dye injections localized vocal motor stimulation sites to regions of the midbrain vocal-acoustic complex. $\boldsymbol{B}$, Biotin injections into the caudal efferent bundle retrogradely filled cells in the auditory efferent nucleus in the rostral medulla. Similar labeling patterns were seen after biotin injections into the saccular efferent bundle (data not shown), verifying that both bundles contain the axons of efferent neurons. LL, Lateral lemniscus; OEC, caudal division of the octavolateralis efferent nucleus; TeM, midbrain tectum; TS, torus semicircularis.

ration of each fictive vocalization is indicated by the gray boxes in Figs. 3A1-C1, 4) (see also Fig. 6A1,B1), with temporal properties (discharge frequency/repetition rate and duration) that closely resembled those of grunts recorded from wild populations of midshipman fish (Bass et al., 1999). Because the animals were immobilized during the experiments, the sonic muscles were inactive; thus, stimulation of mVAC activates the descending vocal motor network without the production of sound (see Materials and Methods). Two bundles of efferent neurons were anatomically distinct from the primary afferent nerves and readily accessible for labeling and neural recordings. Biotin injections into either bundle retrogradely labeled cell bodies in the hindbrain efferent nucleus (Fig. 2 B). Rhodamine and Alexa Fluor injections into the caudal efferent bundle indicated its primary targets to be the lagena and posterior lateral line neuromasts, with a lighter projection to the posterior semicircular canal. This pattern of caudal efferent bundle targets is similar to that seen in the closely related toadfish (Highstein and Baker, 1986). The second bundle 
coursed along the dorsal surface of the saccular afferent nerve, distal to the point at which the saccular nerve joined the main trunk of the auditory nerve to enter the brainstem. Reliable injections into this bundle without spread of tracer into the underlying saccular afferent nerve were difficult, but, based on its position, we are confident that these efferents, like those in toadfish (Highstein and Baker, 1986), project exclusively to the sacculus (for identification of saccular efferent neurons in midshipman after labeling of eighth nerve branches to the sacculus, see Bass et al., 1994). The majority of recordings (59 neurons) were made from the caudal efferent bundle, with additional recordings ( 24 neurons) made from the saccular efferent bundle.

\section{Caudal efferent bundle neurons}

The majority of caudal efferent bundle neurons (51 of 59) showed a change in ongoing activity during the production of fictive vocalizations, with the remaining neurons showing no change in activity. In all cases (including recordings from saccular efferent neurons, see below), neurons only showed a systematic change in activity with glutamate injections that elicited fictive vocalizations. Most of the neurons that showed a response exhibited an increase in activity that occurred near the onset of each fictive vocalization (Fig. $3 A 1, B 1)$, although a subset also showed a decrease in activity near the termination of each fictive vocalization (Fig. 3B1). The remaining responsive neurons showed an overall decrease in activity during fictive vocalizations (Fig. 3C1).

To more thoroughly examine and quantify the nature of the change in efferent activity during fictive vocalizations, we constructed SDFs (see Materials and Methods and Fig. 1) consisting of the mean fictive vocalization activity and mean efferent activity relative to the onset or offset of fictive vocalizations for each neuron (Fig. 3A2,B2,C2). The efferent response was considered to be excitatory if the amplitude of the efferent SDF during fictive vocalization was $>2$ SDs above the mean efferent SDF before fictive vocal onset and suppressive if $<2$ SDs below the mean efferent SDF before fictive vocal onset. Rebound suppression was defined as an efferent SDF at fictive vocalization offset that was $<2$ SDs below the mean efferent SDF after fictive vocal offset.

Using these criteria, the majority (43 of 59) of caudal efferent bundle neurons showed an increase in activity during fictive vocalizations that reached a maximum just after (Table 1, Peak) fictive vocal onset (Fig. 3A2). The duration of excitation was variable (Table 1, Duration) and generally shorter than the mean duration of fictive vocalizations $(82.3 \pm 5.8$, mean \pm SE; $n=43)$.

\section{Excitation/} B1 suppression |

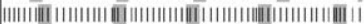

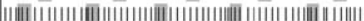

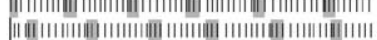

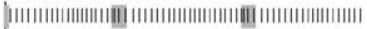

\section{C1 $\begin{gathered}\text { Suppression/ } \\ \text { excitation }\end{gathered}$}

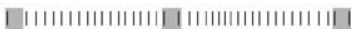

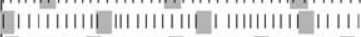

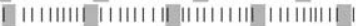

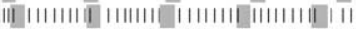
|

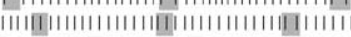

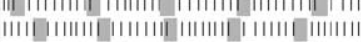

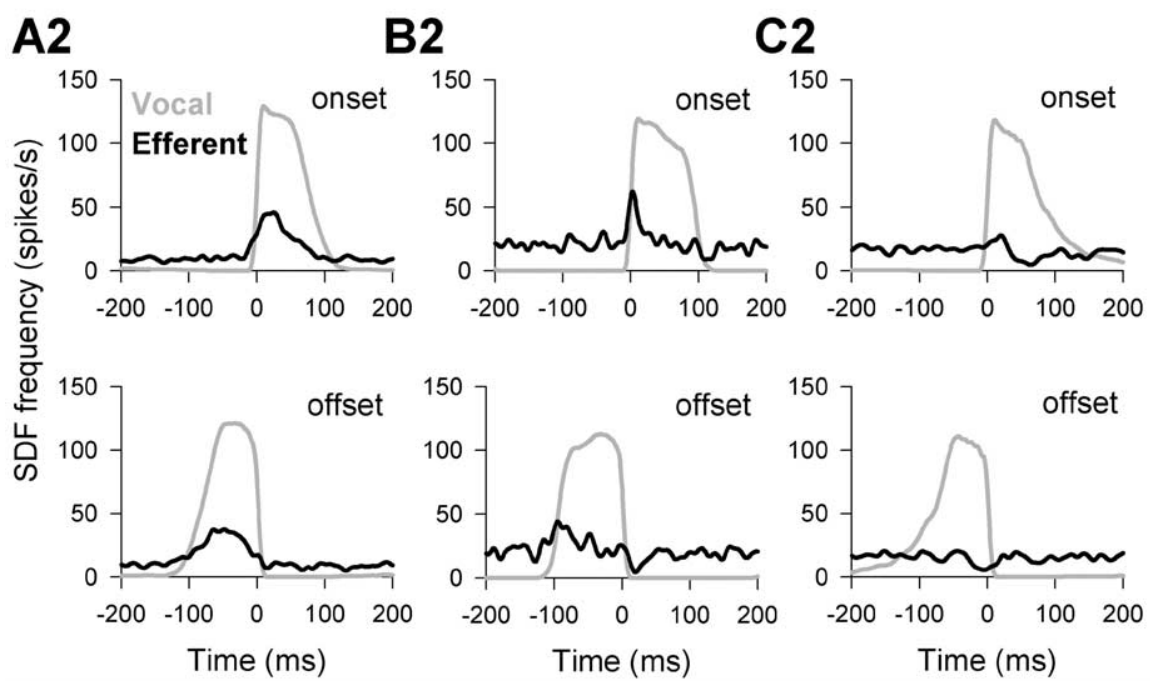

Figure 3. Neuronal activity changes in caudal efferent bundle neurons during fictive vocalizations. Raster diagrams of three representative neurons $(\boldsymbol{A 1}, \boldsymbol{B} \mathbf{1}, \boldsymbol{C} \mathbf{1})$ show raw data of efferent activity (black) during fictive vocalizations (gray), whereas spike density functions $(\boldsymbol{A Z}, \boldsymbol{B} \mathbf{2}, \mathbf{C})$ provide the average efferent activity relative to the onset or offset of fictive vocalizations. Three patterns of efferent activity were evident: $\boldsymbol{A} \mathbf{1}, \boldsymbol{A} \mathbf{2}$, excitation showed increased activity at fictive vocal onset; $\boldsymbol{B} \mathbf{1}, \boldsymbol{B} \mathbf{2}$, excitation/ suppression showed increased activity at fictive vocal onset and decreased activity at fictive vocal offset; $C_{1}, \mathbf{C}$, suppression/ excitation showed suppression of activity during fictive vocalizations that was usually preceded by brief excitation. Scale bar represents time scale for $\boldsymbol{A} \mathbf{1}, \boldsymbol{B} \mathbf{1}, \mathbf{C} \mathbf{1}$.

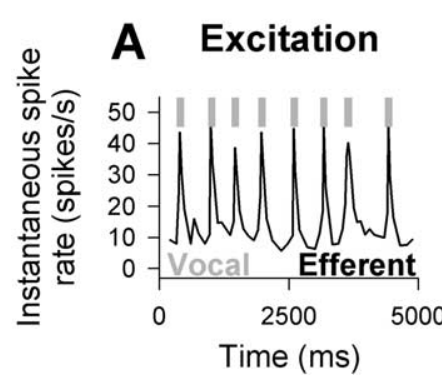

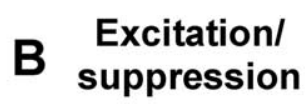

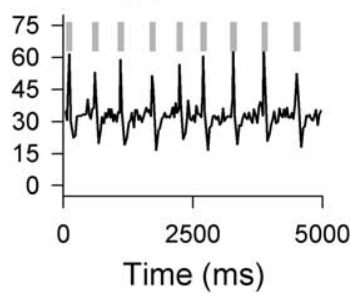

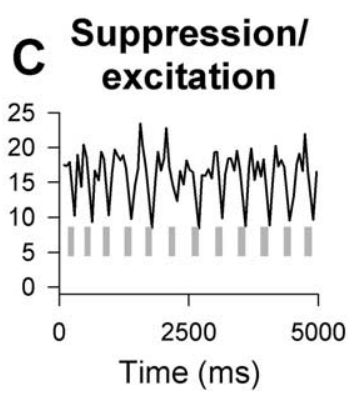

Figure 4. Instantaneous spike rate plots of efferent activity confirmed the patterns shown by spike density functions. $A, B, A s$ shown in these examples, most caudal efferent bundle neurons showed a sharp increase in instantaneous activity at fictive vocalization onset $(\boldsymbol{A})$, with many of these neurons also showing a decrease in activity at fictive vocal offset $(\boldsymbol{B})$. $\boldsymbol{C}, A$ few neurons showed a decrease in instantaneous frequency during fictive vocal activity.

A subset of excitatory neurons (18 of 43) also exhibited a decrease in activity that was correlated with the offset of fictive vocal activity (Fig. 3B2). On average, this rebound suppression was much shorter in duration than the initial excitation (Table 1) and occurred after the termination of each fictive grunt.

A smaller number of neurons (8 of 59) showed suppression, that is, an overall decrease in activity during fictive vocalizations (Fig. 3C2). This change in activity was distinguishable from the rebound suppression after excitation because it was more robustly correlated with the onset, rather than the offset, of fictive vocal activity and occurred during (Fig. 3C2), rather than after, fictive vocalizations (Table 1). As with excitation, the decrease in 
Table 1. Time of maximum activity (Peak) and duration of efferent response measured from spike density functions

\begin{tabular}{|c|c|c|c|c|c|c|}
\hline & \multicolumn{2}{|l|}{ Excitation } & \multicolumn{2}{|c|}{ Rebound suppression } & \multirow{2}{*}{$\begin{array}{l}\text { Suppression } \\
\text { CEB } \\
(n=8)\end{array}$} & \multirow{2}{*}{$\begin{array}{l}\text { Preexcitation } \\
\text { CEB } \\
(n=6)\end{array}$} \\
\hline & $\begin{array}{l}\text { CEB } \\
(n=43)\end{array}$ & $\begin{array}{l}\text { SEB } \\
(n=24)\end{array}$ & $\begin{array}{l}\text { CEB } \\
(n=18)\end{array}$ & $\begin{array}{l}\text { SEB } \\
(n=14)\end{array}$ & & \\
\hline Peak (ms) & $11.7 \pm 2.2$ & $11.8 \pm 1.9$ & $28.4 \pm 6.1$ & $11.0 \pm 3.2$ & $70.4 \pm 10.0$ & $7.0 \pm 5.2$ \\
\hline Duration (ms) & $51.5 \pm 6.6$ & $78.9 \pm 15.7$ & $16.9 \pm 3.0$ & $106.1 \pm 57.1$ & $54.5 \pm 16.2$ & $15.0 \pm 3.0$ \\
\hline
\end{tabular}

Peak was measured relative to fictive vocal onset for excitation, suppression, and preexcitation and relative to fictive vocal offset for rebound suppression. Values are mean \pm SE. CEB, Caudal efferent bundle; SEB, saccular efferent bundle.

been attributable to the longer mean fictive vocal durations $(112.7 \pm 10.4 \mathrm{~ms} ; n=$ 24) during saccular efferent recordings compared with those during caudal efferent bundle recordings $(82.3 \pm 5.8 \mathrm{~ms})$. Fish used in the saccular efferent bundle experiments were collected later during the breeding season than those used in the caudal efferent bundle experiments, which may explain the differences in fictive vocal durations between the two

activity was shorter in duration than the fictive vocalizations but was not restricted to the beginning of fictive vocal activity. In addition, all but two suppressive neurons showed brief excitation immediately preceding the suppression (Fig. 3C2, onset). This excitatory activity was shorter in duration and less robust than the suppressive activity (preexcitation) (Table 1). This is in contrast to the excitatory neurons, in which the excitation was longer and more robust than the rebound suppression.

We constructed plots of instantaneous spike rate (1/interspike interval) for efferent neurons and measured the value of maximum and minimum spike rate (Fig. 4 shows representative examples for each class of neurons). For excitatory neurons, we also measured the time of maximum spike rate relative to fictive vocalization onset and minimum spike rate relative to fictive vocal offset for those neurons showing rebound suppression. For suppressive neurons, we measured the timing of minimum spike rate relative to fictive vocal onset. Excitatory neurons (Fig. $4 A, B$ ) had a mean \pm SE peak rate of $54.9 \pm 5.8$ spikes $/ \mathrm{s}$ that occurred at a latency of $24.4 \pm 3.6 \mathrm{~ms}$ after fictive vocal onset (these values include both excitation and excitation/suppression neurons). The average amplitude of rebound suppression for the excitation/suppression neurons (Fig. $4 B$ ) was $16.0 \pm 2.4$ spikes/s at a latency of $134.8 \pm 29.0 \mathrm{~ms}$ after fictive vocal offset. Suppressive neurons (Fig. 4C) had an average amplitude of $12.8 \pm 1.9$ spikes/s, which occurred at a latency of $160.4 \pm 13.4 \mathrm{~ms}$ after fictive vocal onset.

By increasing the temporal resolution of SDFs (decreasing the width of the Gaussian convolution), we were able to examine the fine temporal properties of the efferent response. More than onehalf ( 22 of 43 ) of the excitatory efferent neurons (includes neurons from both the excitation and excitation/suppression classes) regularly fired action potentials that were tightly phase locked to the individual spikes of the fictive vocalization motor volley (Fig. $5 A$ ). Efferent action potentials lagged the spikes of the fictive vocal motor volley in most (14 of 22) of these neurons (i.e., efferent activity began after the first spike in the vocal motor volley), with latencies ranging from $1.0 \pm 0.71$ to $7.2 \pm 0.73 \mathrm{~ms}$. Activity in the remaining neurons preceded the fictive vocal motor spikes by $0.67 \pm 0.33$ to $3.0 \pm 1.0 \mathrm{~ms}$ (Fig. $5 B$ ). For those neurons that lagged fictive vocal activity, there was a trend toward increased spike latency over the course of a fictive grunt, whereas there was no relationship between spike number and latency in neurons that lead fictive vocal activity (Fig. $5 C$ ).

\section{Saccular efferent neurons}

Unlike caudal efferent bundle neurons, all saccular efferent neurons responded to fictive vocalizations with an increase in activity (Fig. 6A1,B1). This was followed by rebound suppression in 14 of 24 neurons (Fig. 6B1). Like caudal efferent bundle neurons, excitatory activity peaked just after fictive vocal onset, although the mean duration of excitation was longer in saccular efferent neurons (Fig. 6A2; Table 1). The longer excitatory response may have groups. Rebound suppression peaked earlier after fictive vocal offset and was much longer in duration than that for caudal efferent bundle neurons (Fig. 6 B2; Table 1). From instantaneous frequency plots, maximum mean excitatory activity was $71.0 \pm$ 9.7 spikes/s at a latency of $23.4 \pm 5.0 \mathrm{~ms}$ (Fig. 6A3). Rebound suppression rates dropped to $15.8 \pm 3.3$ spikes/s at a latency of $136.9 \pm 28.9 \mathrm{~ms}$ (Fig. 6B3).

Like caudal efferent bundle neurons, the activity of most saccular efferent neurons (16 of 24) was phase locked to the fictive vocalization output (Fig. 7A). However, across saccular efferents, the timing of efferent activity relative to fictive vocal motor activity was more consistent than in caudal efferent bundle neurons. Activity in all but two phase-locked saccular efferent neurons lagged fictive vocal motor activity by $4.0 \pm 0.82$ to $6.0 \pm 0.41 \mathrm{~ms}$ (Fig. $7 B$ ). Unlike the increase in latency observed in caudal efferent bundle neurons that lagged fictive vocal motor activity, there was no systematic change in the latency of saccular efferents over the duration of the fictive vocalizations (Fig. 7C).

\section{Discussion}

We show here a functional link between the vocal motor system and central efferent neurons that innervate the hair cell-based end organs of the inner ear and mechanosensory lateral line in a vertebrate, the plainfin midshipman fish. The majority of efferent neurons exhibited an increase in activity that peaked just after fictive vocalization onset, whereas others showed an overall decrease in activity. This included neurons that target the sacculus and lateral line, two end organs that are sensitive to the frequencies contained within midshipman vocalizations (Weeg and Bass, 2002; Bass and McKibben, 2003). To our knowledge, this is the first direct demonstration of auditory efferent activation by the vocal motor system in any vertebrate. This coupling accomplishes at least three important tasks (Hoy, 2002). First, it tells the animal that an acoustic signal is self-generated; second, it provides timing information to the inner ear and lateral line about when the sound will occur; and third, because saccular and lateral line afferent activity is reduced by efferent activity (Russell, 1971; Russell and Roberts, 1972; Furukawa, 1981; Lin and Faber, 1988), it provides a mechanism whereby peripheral auditory and lateral line sensitivity can be actively reduced during vocalization to prevent overstimulation by reafferent signals.

All saccular efferent neurons responded to fictive vocalizations with an increase in activity correlated with fictive vocal onset, with a subset also showing rebound suppression at fictive vocal offset. The sacculus is the main auditory end organ in most teleosts, including midshipman (Bass and McKibben, 2003). Studies in goldfish show that efferents inhibit saccular afferent activity (Furukawa, 1981). Therefore, the vocal-related increase in efferent activity observed here likely causes a decrease in saccular afferent activity that would effectively decrease auditory sensitivity to self-generated sounds and maintain the sensitivity of hair cells to external sounds by preventing habituation of the 
A
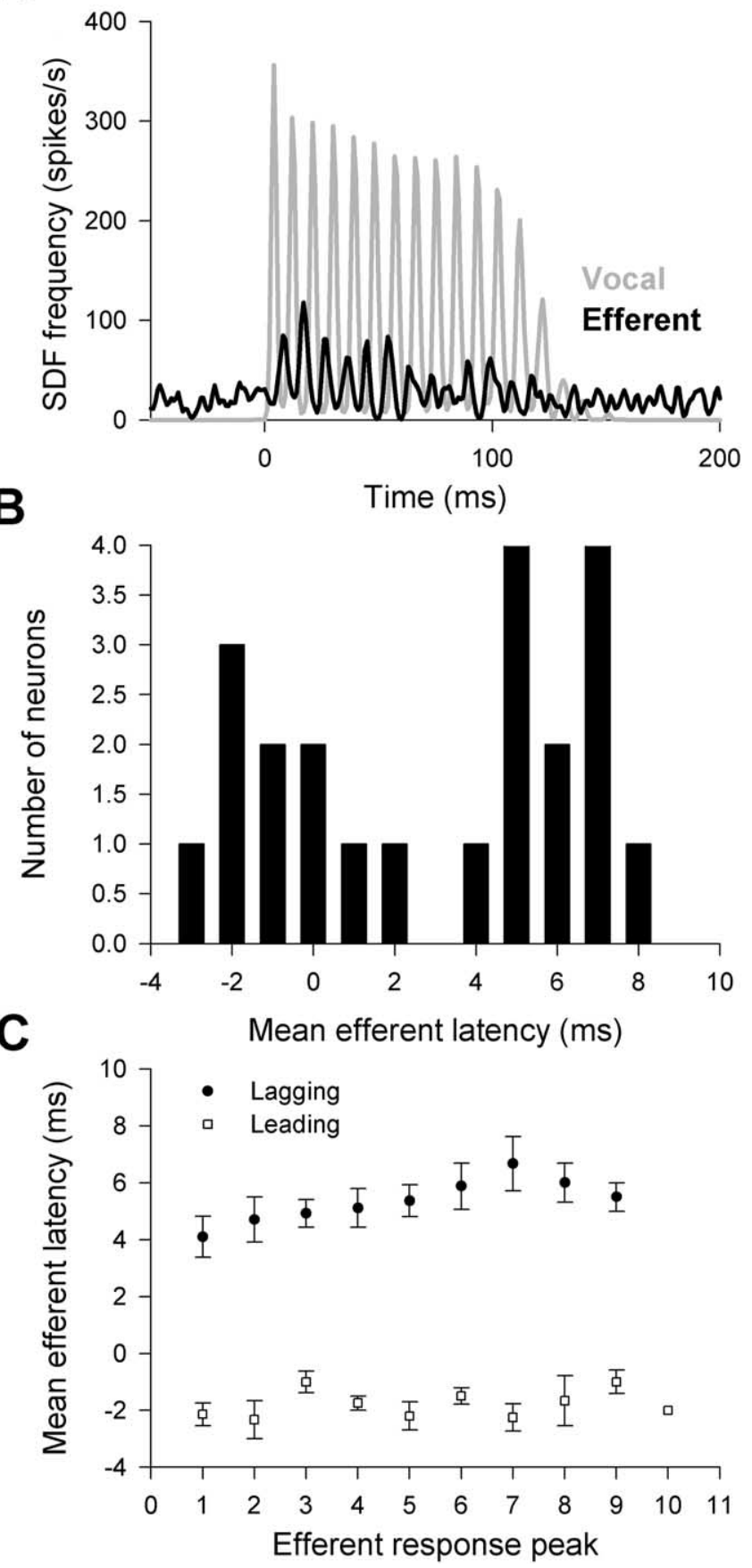

Figure 5. Efferent activity was phase locked to fictive vocal motor output in many caudal efferent bundle neurons. $\boldsymbol{A}$, Spike density functions with high temporal resolution showed that efferent neurons produced peaks in the number of action potentials (black) at precise times relative to the individual compound action potentials that made up the vocal motor response (gray). $\boldsymbol{B}$, The timing of efferent activity was not consistent across neurons, because efferent activity preceded vocal motor output in some neurons and lagged vocal motor activity in other neurons (bin width, $1 \mathrm{~ms})$. C, There was also a slight trend for efferent activity to occur at increasing latencies as the vocal motor output progressed for neurons that lagged vocal motor activity (circles) but not for neurons that lead (squares). Efferent response peak indicates each successive peak in the efferent response during the entire fictive vocalization (see $\boldsymbol{A}$ ).

saccular hair cell-afferent nerve synapse (Furukawa and Matsura, 1978; Furukawa, 1981; Winslow and Sachs, 1987; Lin and Faber, 1988). The rebound suppression may temporarily increase auditory sensitivity immediately after vocalizations. Consistent with
A1

Excitation

B1

Excitation/ suppression
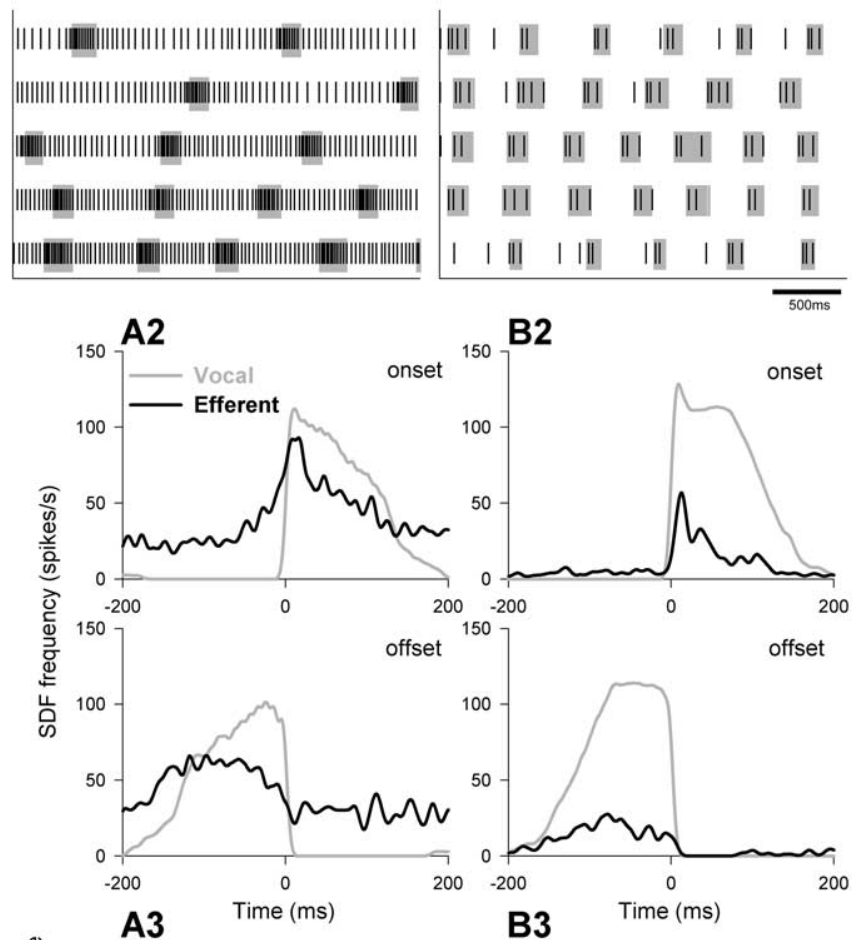

B2
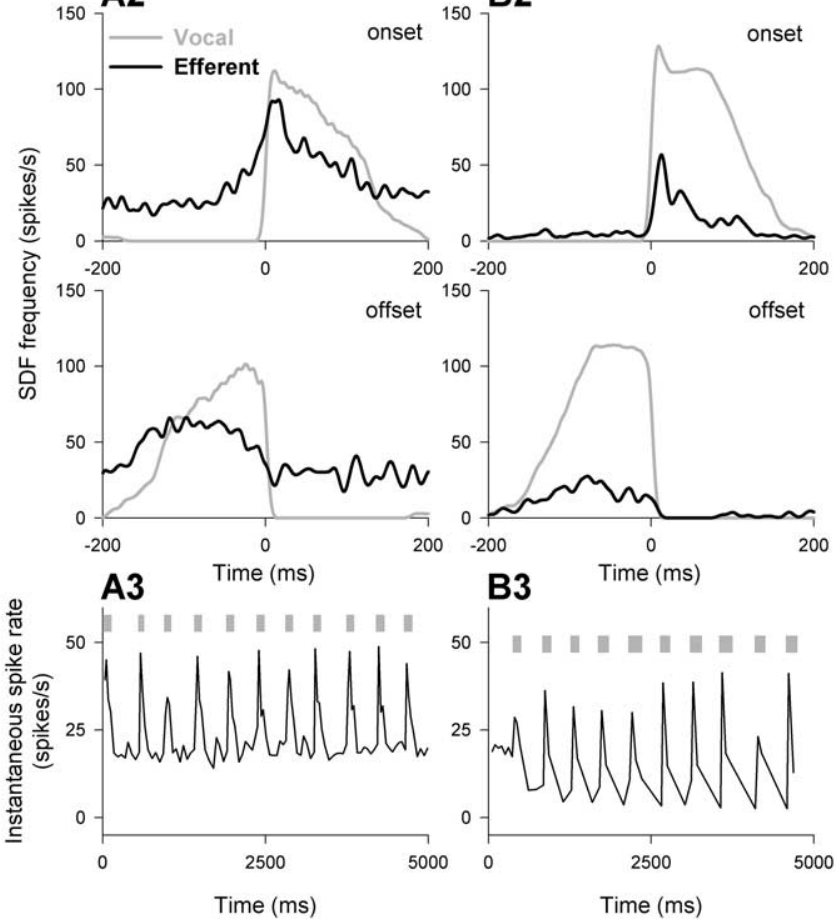

Figure 6. Patterns of saccular efferent bundle activity during fictive vocalizations were similar to those seen in the caudal efferent bundle, except no neurons showed suppression during fictive vocalizations. $\boldsymbol{A}, \boldsymbol{B}$, All saccular efferent neurons (black) exhibited an increase in activity during fictive vocalizations (gray); $\boldsymbol{B}$, in some neurons, this was followed by decreased activity at fictive vocal offset. These responses were evident in raster diagrams $(\boldsymbol{A} 1, \boldsymbol{B} 1)$, spike density functions $(\boldsymbol{A 2}, \boldsymbol{B} 2)$, and plots of instantaneous spike rate $(\boldsymbol{A} \mathbf{3}, \boldsymbol{B} 3)$. Scale bar represents time scale for $A 1, B 1$.

the major role of the sacculus in encoding conspecific vocalizations, this sensitization period lasts much longer in its efferents than those of other end organs.

Caudal efferent bundle neurons showed a greater variety of responses than did saccular efferents. This variability suggests that the efferent system is not a simple modulator but rather consists of multiple components that respond differentially to a given stimulus, which could lead to end organ-specific changes in afferent activity or differential modulation of activity within a single end organ (Tricas and Highstein, 1991). Because the caudal efferent bundle mainly projects to the lagena and posterior lateral line, with a lighter input to the posterior semicircular canal, these different responses may represent efferent neurons that project to different end organs. The posterior lateral line is responsive to a significant portion of the spectral energy of a midshipman call (Weeg and Bass, 2002), whereas the lagena is an otolithic end organ that may serve an auditory function in a limited capacity (Lu et al., 2003). Thus, the posterior lateral line and lagena would both be likely targets of excitatory efferent neurons that would 


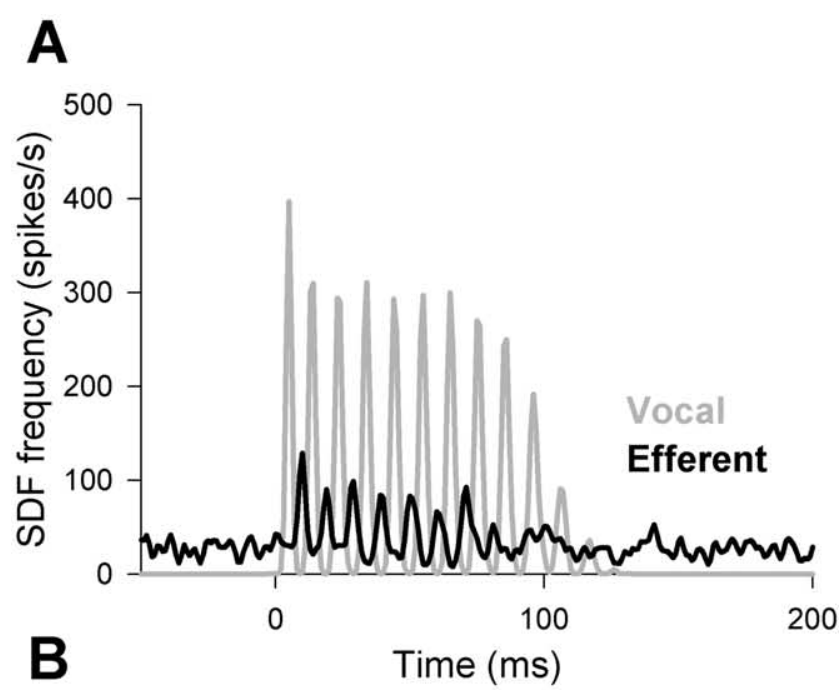

be the target of suppressive efferent neurons, which should decrease vestibular activity (Highstein and Baker, 1985; Boyle and Highstein, 1990). Alternatively, it may not be necessary to modulate vestibular sensitivity during vocalization, and the posterior semicircular canal may be the target of unresponsive efferent neurons.

The temporal periodicity of efferent responses, especially for saccular efferents, reflects the precise timing information that is inherent to the vocal pattern-generating network that initiates the rhythmic, oscillatory-like firing of sonic motor neurons (Bass and Baker, 1990). This could have important implications for the temporal nature of afferent modulation. Midshipman saccular and lateral line afferents respond to tonal stimuli in a phaselocked manner, firing action potentials only during part of the stimulus waveform (McKibben and Bass, 1999; Weeg and Bass, 2002; Weeg et al., 2002). If the timing of efferent activity matches the phase at which its target afferent responds to a reafferent vocalization, afferent sensitivity could be modulated only during a specific portion of the vocal waveform and remain unchanged during the remainder. This could be a finely tuned temporal mechanism by which sensitivity to reafferent stimuli is reduced while sensitivity to external sounds is essentially maintained.

The fictive vocalizations induced by our experimental paradigm are similar in duration and pulse repetition rate to agonistic grunts produced by midshipman males (Bass et al., 1999). Hums, which are advertisement calls, are much longer (up to $1 \mathrm{~h}$ ) and are difficult to elicit in a physiological preparation. Given the duration of the efferent response, the midshipman efferent system may be maximally suited for adjusting auditory sensitivity at the onset and offset of vocalizations. It should be noted that we report the timing of the efferent response relative to the timing of the vocal motor volley recorded from the sonic nerve. Because sonic muscle contraction occurs $\sim 5 \mathrm{~ms}$ after sonic nerve activity (Packard, 1960; Skoglund, 1961; Bass and Baker, 1990), the timing of the efferent response relative to sound production will be shifted by this amount. However, this should be a uniform shift across all efferent neurons and does not affect the interpretation of our results. Moreover, because saccular efferent activity on average lagged fictive vocal activity by $4-6 \mathrm{~ms}$, it should closely concur with the onset of an actual vocalization in vivo.

Although the physiological effects of efferent stimulation on afferent activity are well established (Art et al., 1982; Roberts and Meredith, 1992), the behavioral relevance of the efferent system is not well understood. Lateral line efferent activity increases during movement in frogs (Russell, 1971) and dogfish (Roberts and Russell, 1972), whereas behavioral arousal causes an increase in efferent activity that is accompanied by a decrease in lateral line afferent activity in toadfish (Tricas and Highstein, 1990, 1991). Based on these results, it is hypothesized that the efferent system functions to decrease the response of the lateral line to selfgenerated water motions, such as those caused by swimming or respiratory movements (Tricas and Highstein, 1991). It is likely that vocal-related increases in efferent activity reported here similarly lead to decreased auditory and lateral line afferent activity. Several features of saccular efferents seem especially well adapted to maintaining sensitivity to conspecific and other acoustic signals both during and immediately after sound production. First, as discussed previously, the especially robust phase locking of saccular efferents to each cycle of the fine structure of a vocalization provides a mechanism for maintaining sensitivity to concurrent vocalizations from conspecifics, which is a natural listening task for midshipman especially when producing their advertisement calls (Bass et al., 1999). Second, the early onset and long

reduce sensitivity of these end organs in a similar manner to the sacculus. Consistent with an overall decrease in inner ear activity during vocalizations, posterior semicircular canal afferents may

Figure 7. Saccular efferent neuronal activity was phase locked to vocal motor output in many neurons. $A$, Spike density functions with high temporal resolution showed that efferent neurons produced action potentials (black) at precise times relative to the individual compound efferent activity was more uniform than caudal efferent bundle activity, with only two neurons esaling vocal motor output. C, There was no trend for either lagging (circles) or leadin (squares) efferent activity to occur at increasing latencies as the vocal motor output progressed. 
duration of the rebound suppression of saccular efferents provides a rapid and relatively long-lasting period of sensitization for the detection of acoustic stimuli.

Considering the extensive similarities in the pattern of auditory brainstem organization of teleosts to that of other vertebrates (McCormick, 1999; Bass et al., 2005), including efferents to the inner ear (Roberts and Meredith, 1992; Manley, 2000), we propose that the vocal motor-efferent coupling identified here provides a mechanism for all sonic vertebrates to decrease peripheral auditory sensitivity during sound production. Although such a mechanism may be especially important in vertebrates that lack a middle ear response, it may also supplement the middle ear response by decreasing sensitivity of the cochlea to stimulation via bone conduction, which would not be affected by the middle ear response (Suga and Jen, 1975). Although cochlear microphonic potentials in bats are not consistent with auditory efferent activation during vocalization (Suga and Jen, 1975), cochlear microphonic decay times during vocalization are suggestive of an efferent effect (Goldberg and Henson, 1998). The presence of a shared mechanism of vocal motor-auditory efferent coupling between such distantly related groups as teleosts and tetrapods would be a striking example of convergent evolution among vertebrates that need to solve similar sensory reafference problems.

\section{References}

Art JJ, Crawford AC, Fettiplace R, Fuchs PA (1982) Efferent regulation of hair cells in the turtle cochlea. Proc R Soc Lond B Biol Sci 216:377-384.

Bass AH, Baker R (1990) Sexual dimorphisms in the vocal control system of a teleost fish: morphology of physiologically identified neurons. J Neurobiol 21:1155-1168.

Bass AH, McKibben JR (2003) Neural mechanisms and behaviors for acoustic communication in teleost fish. Prog Neurobiol 69:1-26.

Bass AH, Marchaterre MA, Baker R (1994) Vocal-acoustic pathways in a teleost fish. J Neurosci 14:4025-4039.

Bass AH, Bodnar DA, Marchaterre MA (1999) Complementary explanations for existing phenotypes in an acoustic communication system. In: Neural mechanisms of communication (Hauser M, Konishi M, eds), pp 493-514. Cambridge, MA: MIT.

Bass AH, Rose GJ, Pritz MB (2005) Auditory midbrain of fish, amphibians and reptiles: model systems for understanding auditory function. In: The inferior colliculus (Winer JA, Schreiner CE, eds), pp 459-492. New York: Springer.

Boyle R, Highstein SM (1990) Efferent vestibular system in the toadfish: action upon horizontal semicircular canal afferents. J Neurosci 10:1570-1582.

Furukawa T (1981) Effects of efferent stimulation on the saccule of goldfish. J Physiol (Lond) 315:203-215.

Furukawa T, Matsura S (1978) Adaptive rundown of excitatory postsynaptic potentials at synapses between hair cells and eighth nerve fibers in the goldfish. J Physiol (Lond) 276:193-209.

Goldberg RL, Henson OW (1998) Changes in cochlear mechanics during vocalization: evidence for a phasic medial efferent effect. Hear Res 122:71-81.

Goodson JL, Bass AH (2002) Vocal-acoustic circuitry and descending vocal pathways in teleost fish: convergence with terrestrial vertebrates reveals conserved traits. J Comp Neurol 448:298-322.

Grassi S, Magni F, Ottaviani F (1988) Mechanisms controlling vocalizationevoked stapedius muscle activity in chickens (Gallus gallus). J Comp Physiol 162:525-532.

Highstein SM, Baker R (1985) Action of the efferent vestibular system on primary afferents in the toadfish, Opsanus tau. J Neurophysiol 54:370-384.

Highstein SM, Baker R (1986) Organization of the efferent vestibular nuclei and nerves of the toadfish, Opsanus tau. J Comp Neurol 243:309-325.

Hoy R (2002) Neurobiology: tuning in by turning off. Nature 418:831-832.

Lin JW, Faber DS (1988) An efferent inhibition of auditory afferents mediated by the goldfish Mauthner cell. Neuroscience 24:829-836.

Lu Z, Xu Z, Buchser WJ (2003) Acoustic response properties of lagenar nerve fibers in the sleeper goby, Dormitator latifrons. J Comp Physiol 189:889-905.

Manley GA (2000) Cochlear mechanisms from a phylogenetic viewpoint. Proc Natl Acad Sci USA 97:11736-11743.

McCormick CA (1999) Anatomy of the central auditory pathways of fish and amphibians. In: Comparative hearing: fish and amphibians (Fay RR, Popper AN, eds), pp 155-217. New York: Springer.

McKibben JR, Bass AH (1999) Peripheral encoding of behaviorally relevant acoustic signals in a vocal fish: single tones. J Comp Physiol 184:563-576.

Packard A (1960) Electrophysiological observations on a sound-producing fish. Nature 187:63-64.

Paulin MG (1992) Digital filters for firing rate estimation. Biol Cybern 66:525-531.

Popper AN, Fay RR (1999) The auditory periphery in fishes. In: Comparative hearing: fish and amphibians (Fay RR, Popper AN, eds), pp 43-100. New York: Springer.

Poulet JFA, Hedwig B (2002) A corollary discharge maintains auditory sensitivity during sound production. Nature 418:872-876.

Roberts BL, Meredith GE (1992) The efferent innervation of the ear: variations on an enigma. In: The evolutionary biology of hearing (Webster DB, Fay RR, Popper AN, eds), pp 185-210. New York: Springer.

Roberts BL, Russell IJ (1972) The activity of lateral-line efferent neurons in stationary and swimming dogfish. J Exp Biol 57:435-448.

Russell IJ (1971) The role of the lateral-line efferent system in Xenopus laevis. J Exp Biol 54:621-641.

Russell IJ, Roberts BL (1972) Inhibition of spontaneous lateral-line activity by efferent nerve stimulation. J Exp Biol 57:77-82.

Skoglund CR (1961) Functional analysis of swim-bladder muscles engaged in sound production in toadfish. J Biophys Biochem Cytol 10:187-200.

Suga N, Jen PHS (1975) Peripheral control of acoustic signals in the auditory system of echolocating bats. J Exp Biol 62:277-311.

Szucs A (1998) Applications of the spike density function in analysis of neuronal firing patterns. J Neurosci Methods 81:159-167.

Tricas TC, Highstein SM (1990) Visually mediated inhibition of lateral line primary afferent activity by the octavolateralis efferent system during predation in the free-swimming toadfish Opsanus tau. Exp Brain Res 83:233-236.

Tricas TC, Highstein SM (1991) Action of the octavolateralis efferent system upon the lateral line of free-swimming toadfish, Opsanus tau. J Comp Physiol 169:25-37.

von Holst E, Mittelstaedt H (1950) Das Reafferenzprinzip. Naturwissenschaften 37:464-476.

Weeg MS, Bass AH (2000) Central lateral line pathways in a vocalizing fish. J Comp Neurol 418:41-64.

Weeg MS, Bass AH (2002) Frequency response properties of lateral line superficial neuromasts in a vocal fish, with evidence for acoustic sensitivity. J Neurophysiol 88:1252-1262.

Weeg MS, Fay RR, Bass AH (2002) Directionality and frequency tuning of primary saccular afferents of a vocal fish, the plainfin midshipman (Porichthys notatus). J Comp Physiol 188:631-641.

Winslow RL, Sachs MB (1987) Effect of electrical-stimulation of the crossed olivocochlear bundle on auditory-nerve response to tones in noise. J Neurophysiol 57:1002-1021.

Yost WA (2000) Fundamentals of hearing: an introduction, Ed 4. San Diego: Academic. 